\title{
Building feature extraction method based on double reflection imaging dictionary
}

\author{
Honggao Deng ${ }^{1,2}$, Tingfa $\mathrm{Xu}^{1 *}$, Qinghua Liu ${ }^{2}$ and Yuhan Zhang ${ }^{1}$
}

\begin{abstract}
In the technology of building feature extraction, multi-path virtual image appears in the imaging result because the imaging scene is composed of complex wall model and the observation distance of the through-wall radar is short. In order to solve this problem, this paper proposes a double reflection imaging dictionary algorithm to extract building scattering center. In this algorithm, the propagation models of direct path and double reflection path are established with the direct path imaging dictionary and the double reflection imaging dictionary, the corresponding optimization problems are constructed. Finally, the multi-perspective fusion imaging steps are taken to suppress the false image according to the non-correlation of false image from the different reflection path. Experimental results verify the effectiveness of the proposed algorithm.
\end{abstract}

Keywords: Through-the-wall radar imaging (TWRI), The direct path imaging dictionary, The double reflection imaging dictionary, Building dominant scatterers

\section{Introduction}

UWB through-wall-radar imaging (TWRI) is a new technology in recent years, which can obtain high resolution images of buildings all day [1]. TWRI technology can determine the layout of buildings and identify indoor location information, which has wide practical value in disaster relief, counter-terrorism operations and fire protection [2]. At present, most TWRI systems observe the building's wall imaging by multi view [3, 4], and the layout of the building is achieved by the radar image fusion method. This treatment will cause that the layout of the buildings is not intuitive enough, and the walls cannot match each other with lots of image thorn. A few TWRI systems use the electromagnetic characteristics of scatterers to extract the main scattering objects from the wall echo data, and get clear and complete layout images by graph theory, and overcome the influence of clutter and multipath [5-7]. This paper also focuses on this type TWRI system.

Most of the energy in the building scene is only provided by a few strong scattering centers, which are corners, indicating that the parameter space of radar

\footnotetext{
*Correspondence: ciom_xtf@bit.edu.cn

${ }^{1}$ Key Laboratory of Photoelectronic Imaging Technology and System, Beijing Institute of Technology, Beijing, China

Full list of author information is available at the end of the article
}

echo in the corner scattering center is of high sparsity $[8,9]$. Therefore, building corners can be extracted by compressive sensing theory. In recent years, some research institutions have applied the compressive sensing theory to the TWRI system, and proposed many practical and efficient imaging algorithms. They divided the building scene into multiple grid points, assuming that the corner can be located at any grid point of the scene, and achieved the better imaging effect [4-7].

In [4], a ghosting suppression method based on corner azimuth features is proposed. This method fuses multiple sub-aperture images with full-aperture images to eliminate multiple aperture ghosting. However, a large number of antenna arrays should be set around buildings, and the performance of this method is greatly influenced by the number and orientation of the antenna array. In practical application, it is difficult to select the proper azimuth of the sub-aperture and the number of electromagnetic wave data acquisition is huge, so the method is not practical.

In order to reduce the number of data collection and time, and increase the scattering signal in the corner of the building, oblique illumination is considered, which specially enhances the radar returns from the corners formed by the orthogonal intersection of two walls [5-7]. In [7], an oblique MIMO radar antenna array is used to 
reduce the sidelobe of the corner, and the layout of the building through the corner location information is indirectly obtained. However, the wall compensation step is employed to compensate for the delay of the wall parallel to the antenna array, which is not applicable to the oblique antenna array, resulting in the deviation of corner position. In [6,7], the influence of azimuth of antennas on building scatterers is considered, and an over complete dictionary based on antenna azimuth variables is proposed, which can completely restore location information of main scatters. However, the method in [7] considers the corner of a building as a simple point target, while the corner should be regarded as an extended target when detecting the building in the near field. This method fails to take into account the dihedral angle attribute of the corner and ignores the double reflection phenomenon of the corner echo, resulting in defocus, background noise, and more wall clutter in the corner imaging.

When using through-wall radar test building, the phenomenon of multipath effect exists in the actual environment. Antenna array receives not only the corner direct reflection echo signal, but also multipath transmission signals from the wall reflection and the second reflection signals from the corner. If the information contained in the double reflection wave of the corner is used as the supplement of the corner information, the false image can be effectively suppressed while the signal strength of the corner is strengthened.

From this point of view, in this paper, a combined algorithm based on direct wave dictionary and secondary wave dictionary is proposed to extract the scattering center of buildings and remove the multi-path ghost. Firstly, the corner is regarded as the extended target, and the direct path model and the double reflection model are constructed based on corner characteristics. Each path is regarded as an observation channel and concentrated on the same compressed sensing model. Then the direct path imaging dictionary and the double reflection imaging dictionary are constructed, and the single view imaging is obtained by using the compressed sensing method. Finally, the multi-perspective fusion imaging is used to suppress the false image. The method can not only make full use of the double reflection information of the corner to accurately extract the scattering center of the building, but also use the non-correlation of false image from the different reflection path to suppress a large number of false images and noises. The simulation and real data are used to prove the validity of the proposed approach.

This method can not only make full use of the information of corner echo to accurately extract the scattering center of buildings, but also use the false image noncorrelation of different observation models to suppress a large number of false images and noises. Simulation and experimental results verify the effectiveness and feasibility of the proposed method.

The rest of the paper is organized as follows. In Section II, the direct path model and the double reflection model are introduced. In Section III and Section IV, the detailed processing steps of the proposed imaging dictionary and image fusion algorithm are described. In Section V, the GprMax simulation data and the real experimental data are provided to evaluate the performance of the proposed algorithm. The conclusions are drawn in Section VI.

\section{Results}

In this section, several computer simulation and real data collection experiment results are provided to validate the proposed algorithm.

\subsection{Simulation results}

As shown in Fig. 1a, $2.00 \mathrm{~m} \times 2.00 \mathrm{~m}$ square building model is established including six walls and four corners, the walls are $0.10-\mathrm{m}$ thick and made of solid concrete with permittivity of 4.5 . The transceiver array is $2.00 \mathrm{~m}$ from the corner of the building, and the antenna array is oblique to the walls, as shown in Fig. 1. The simulated data is obtained using GprMax simulation. A steppedfrequency signal consisting of 201 frequencies covering the frequency band of $1-2 \mathrm{GHz}$ was used for interrogating the scene. The inter-element spacing of sensors is $0.10 \mathrm{~m}$. In this simulation, we study the performance of the proposed algorithm for the case when only the path $L_{1}$ exist. In the unknown position of the corner, we assume that the corners can be located at any pixel of the image and all corners have the same orientation angle, which is determined by the oblique illumination under consideration [7], so we set the orientation angle of the scatterers $\bar{\phi}_{p} \approx 0^{\circ}$ [9] and angular tilt of the array $\phi_{p, n} \in\left[0^{\circ}, 90^{\circ}\right]$. The oblique antenna array is used to re-



Fig. 1 The simulation scenario 
duce the echo of the wall. In this simulation, the angular tilt of the array baseline is $45^{\circ}$, we can get better results when the tilt angle is set to the range of $\left[30^{\circ}, 60^{\circ}\right]$.

The scene grid is meshed by interval $0.10 \mathrm{~m} \times 0.10 \mathrm{~m}$, and the discrete observation equation of radar received signal is derived based on the direct propagation path model and the double reflection propagation path model. Imaging result considering only the direct path is shown in Fig. 2. The image with respect to only three double reflection imaging dictionary is shown in Fig. 3.

The image fusion result is shown in Fig. 4. It is clear that the multipath noises have been deleted completely and the location and strength of the corner can be detected correctly from Fig. 4. Using the algorithms of [7], although the corner location can be roughly identified, there exists the phenomenon of defocus, noise, and wall clutter shown in Fig. 5.

In order to more directly analyze the performance of the proposed algorithm, three evaluation criteria of target-to-clutter ratio (TCR), signal-to-clutter ratio (SCR), and entropy of the image (ENT) are provided in Table 2. The TCR value of an image is defined as the ratio between the highest pixel intensity value of the true corner location area to the maximum pixel intensity value of the clutter area. The higher the TCR value, the clearer the target is relative to the background clutter [14]. The ENT value of an image determines the complexity of imaging, and the ENT value is smaller when the whole graph is less clutter. The SCR value of an image is the ratio of the power in the target regions to the power summation of the rest regions. When the background noise is more fully suppressed and the quality of the imaging results is better, the SCR is larger.

In Table 1, the ENT value of [7] is larger, and the clutter of the image is much more. We observe that the TCR value and the SNR value of this method are larger,

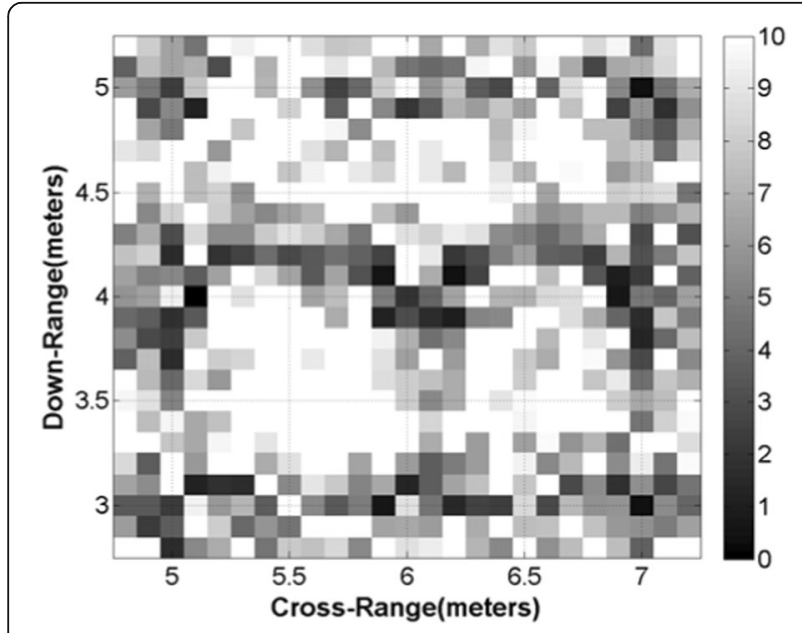

Fig. 2 The image from direct path



Fig. 3 The image from double reflection path

the target relative background clutter is stronger, the background noise is suppressed sufficiently, and the quality of the imaging results is better, thus improving the detection performance.

\subsection{Experimental results}

In order to verify the reconstruction effect of the proposed algorithm in the actual radar data, a radar test system was constructed by using vector network analyzer and horn antenna. The system configuration diagram and the measured scene are shown in Fig. 6. The experimental scene geometry is depicted in Fig. 7. The thickness of the wall is $0.20 \mathrm{~m}$ and relative permittivity of 6.4 . The width of the front wall and the side walls are $2.45 \mathrm{~m}$ and $2.40 \mathrm{~m}$, respectively. This radar test system is placed $1.00 \mathrm{~m}$ from the corner of the building. Four sets of experimental data are tested in the experiment with a sample shown in Fig. 7. The tilt angular of the array baseline is $45^{\circ}$. The radar

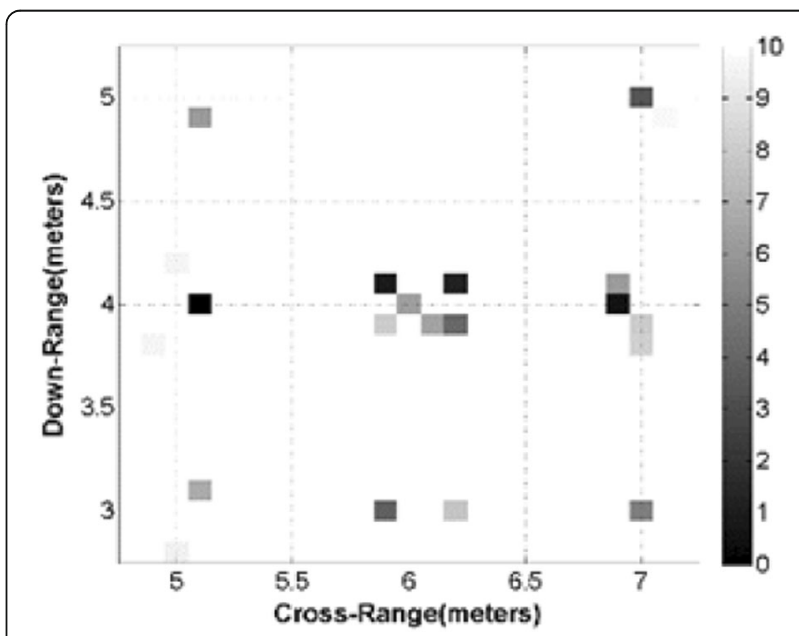

Fig. 4 The fusion result 


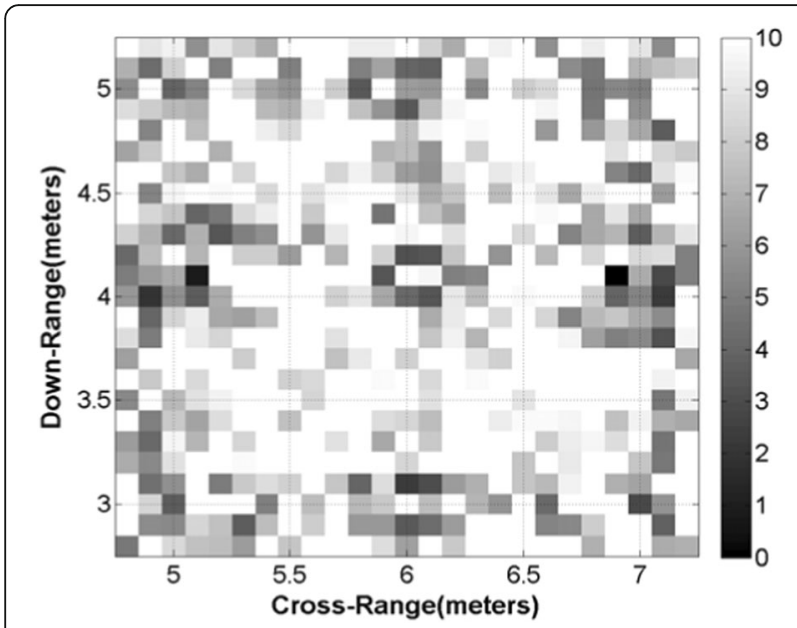

Fig. 5 The image result of [7]

antenna installed on the car forms a $1.80 \mathrm{~m}$ long synthetic aperture, and the interval between adjacent antennas is $0.10 \mathrm{~m}$. The stepped frequency continuous waveform is employed [15], with the frequency starting from $1 \mathrm{GHz}$ to $2 \mathrm{GHz}$ with the frequency interval $10 \mathrm{MHz}$.

The multi-viewpoint imaging results of direct path imaging dictionary and double reflection imaging dictionary are shown in Figs. 8 and 9, respectively. The final fusion result is shown in Fig. 10. It is clear that the noises have been deleted completely and the location and strength of the corner can be detected correctly. As shown in Fig. 11 with the algorithms of [7], although the corners of interest are visible in the image, it is difficult to discriminate them from other scatterers and clutter.

As can be seen from Table 2, the ENT values of [7] are larger, the clutter of images is more, and the images are relatively chaotic. At the same time, the TCR and SCR values of the proposed algorithm are larger, and the quality of imaging results is better.

\section{Conclusions}

This paper considers the problem of multipath ghosts elimination for the through-wall radar imaging. In the experiment of building feature extraction, while the observation distance between radar and building is relatively closer, the corner of a building is regarded as a complex scatter. So if the corner is regarded as a simple point target to be extracted, the image will appear defocus and more false images. In order to solve this problem, the double reflection imaging dictionary is proposed to extract

Table 1 Comparison of effect of simulation experiment

\begin{tabular}{llll}
\hline Algorithm & $\mathrm{TCR} / \mathrm{dB}$ & ENT & $\mathrm{SCR} / \mathrm{dB}$ \\
\hline The algorithm in [7] & 1.6843 & 0.6518 & -10.0881 \\
The proposed algorithm & 2.4852 & 0.1157 & -0.2118 \\
\hline
\end{tabular}

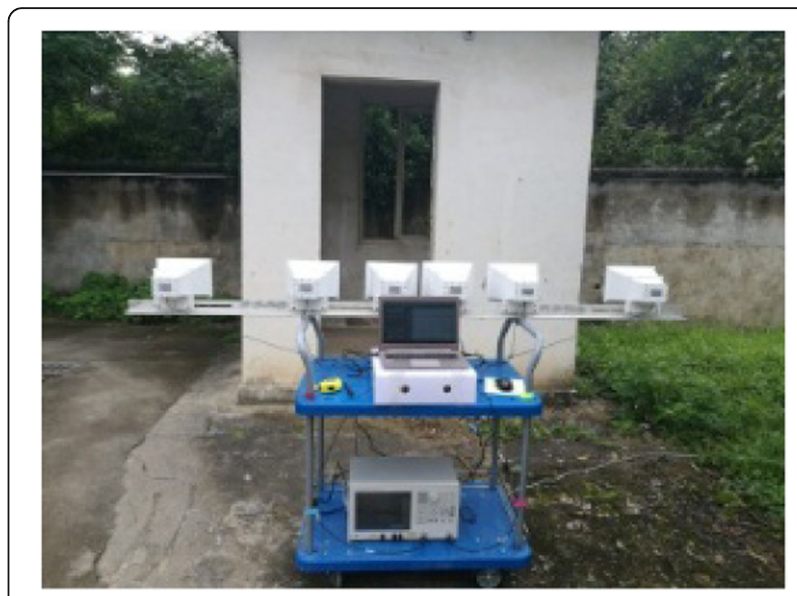

Fig. 6 Photograph of the experimental scene

the scattering center of the building by building a direct wave dictionary and the second reflection dictionary. Multi-perspective image fusion method is used to accurately identify the corner position of the building area, enhance the scattering amplitude of the corner, suppress a lot of wall clutter and background noise. The processing results of GprMax simulation data and experimental data show that the whole image is relatively clear.

\section{Methods}

\subsection{TWRI signal model}

As shown in Fig. 12, the building model is a simple layout of three rooms with the walls of $0.1 \mathrm{~m}$ thickness. We consider a monostatic synthetic aperture radar imaging system. The data acquisition is carried out in an oblique position, which significantly attenuates the wall returns and enhances corner scatterers. Let the antenna at the $n$th location illuminates the scene with a steppedfrequency signal of $M$ frequencies, and the $m$ th frequency $\omega_{m}$ is defined as

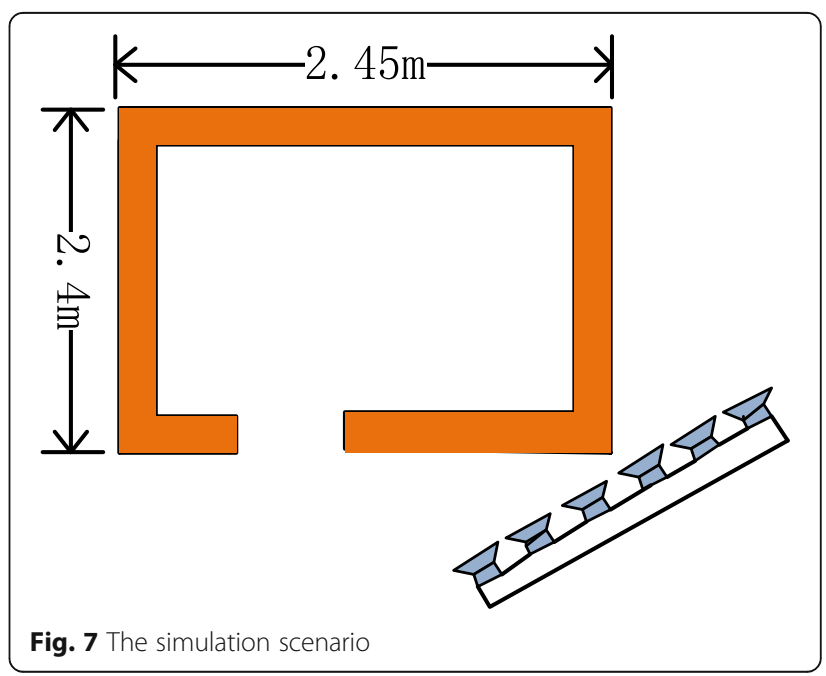




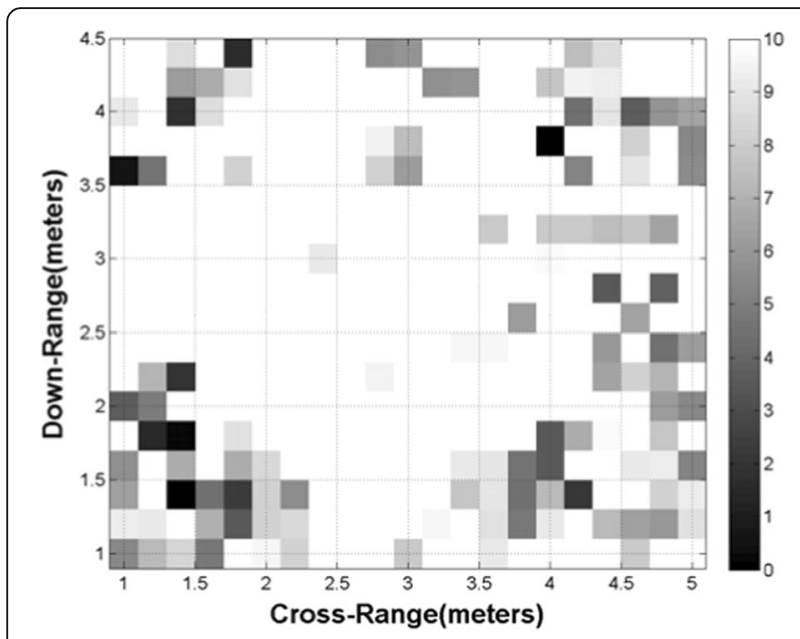

Fig. 8 The image from direct path

$$
\omega_{m}=\omega_{0}+m \Delta_{\omega}, m=0, \cdots, M-1
$$

where $\omega_{0}$ and $\Delta_{\omega}$ denote the lowest frequency in the bandwidth spanned by the stepped-frequency signal and the frequency step size, respectively.

\subsection{The direct propagation path signal model}

According to the theory of physical optics, the response of the scene can be modeled as the sum of responses from individual corner scatterers, assuming that the scatterers do not interact with each other. Thus, the direct path echo received by the $n$th transceiver at the $m$ th frequency can be represented as $[6,7]$

$$
\begin{aligned}
y_{T}(m, n)= & \sum_{p=1}^{Q} S_{p}\left(m, n, \bar{\phi}_{p}\right) \exp \left(-j \omega_{m} \tau_{p, n}^{(A)}\right) \\
& +w(m, n)
\end{aligned}
$$

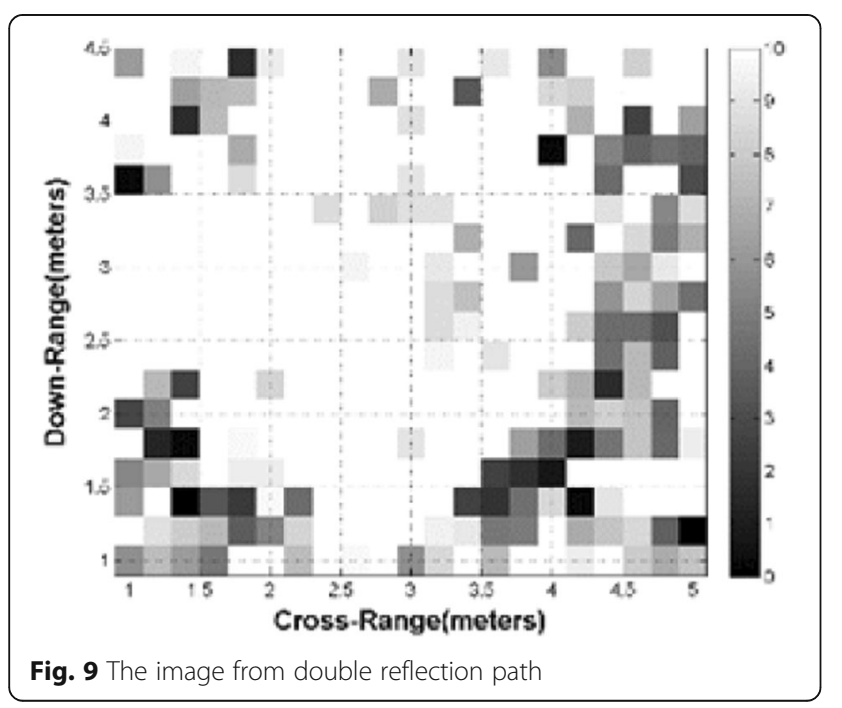

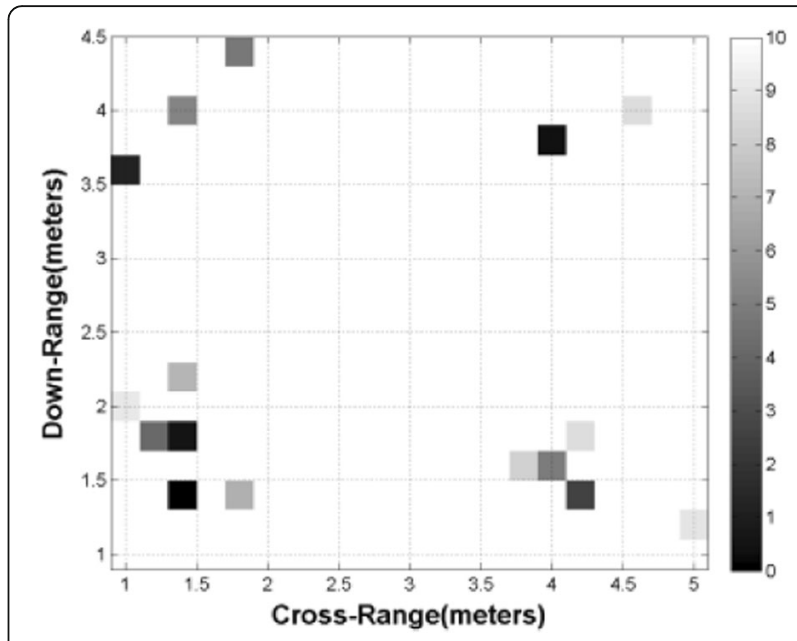

Fig. 10 The fusion result

where $Q$ is the number of corner scatterers present in the illuminated scene, $\tau_{p, n}^{(A)}$ is the direct path two-way traveling time of the signal from the $n$th antenna to the $p$ th corner scatterer. The term $w(m, n)$ in Eq. (2) models the contributions of other wall scatterers, noise, and the multipath propagation effects. The canonical scattering response $S_{p}\left(m, n, \bar{\phi}_{p}\right)$ of the $p$ th corner reflector is given by [7]

$$
S_{p}\left(m, n, \bar{\phi}_{p}\right)=A_{p} \sin c\left(\frac{\omega_{m} L_{p}}{c} \sin \left(\phi_{p, n}-\bar{\phi}_{p}\right)\right)
$$

where the variables $A_{p}, L_{p}$, and $\bar{\phi}_{p}$, respectively, define the amplitude, length, and orientation angle of the $p$ th corner, and the variable $\phi_{p, n}$ denotes the aspect angle associated with the $p$ th corner reflector and the $n$th antenna. $c=3 \times 10^{8} \mathrm{~m} / \mathrm{s}$.

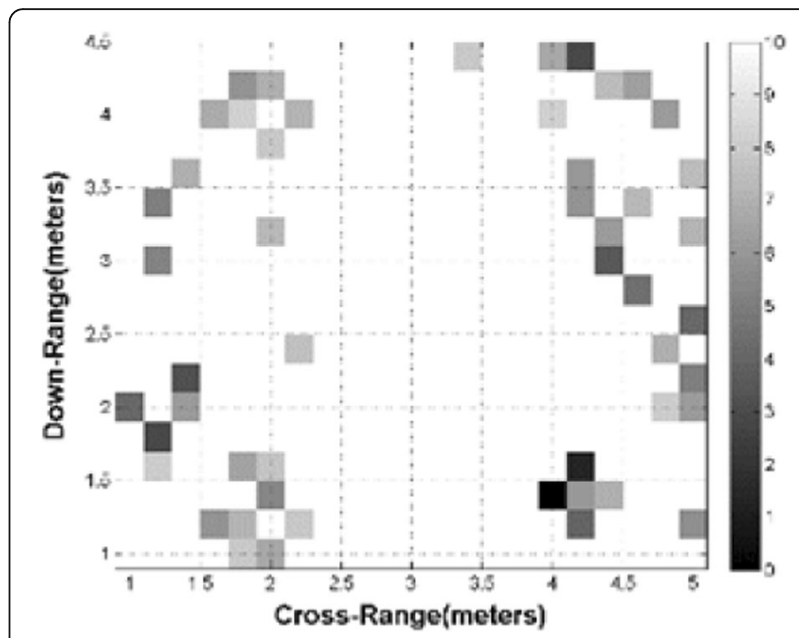

Fig. 11 The image of [7] 
Table 2 Comparison of the effect of measured algorithm

\begin{tabular}{llll}
\hline Algorithm & TCR/dB & ENT & SCR/dB \\
\hline The algorithm in [7] & 1.0288 & 0.4774 & -8.2636 \\
The proposed algorithm & 2.1964 & 0.2221 & -4.9635 \\
\hline
\end{tabular}

When the antenna array obliquely illuminates the wall, the echo transmission path is shown in Fig. 13a, and the direct path traveling time of the signal from the $n$th antenna to the $p$ th corner scatterer can be represented as

$$
\begin{aligned}
\tau_{p, n}^{(A)} & =\frac{2 d_{0}}{c}+\frac{2 d(\sqrt{\varepsilon}-1)}{c \cdot \cos \theta} \\
& =\frac{2 \sqrt{\left(x_{p}-x_{n}\right)^{2}+\left(y_{p}-y_{n}\right)^{2}}}{c}+\frac{2 d(\sqrt{\varepsilon}-1)}{c \cdot \cos \theta}
\end{aligned}
$$

where $\left(x_{p}, y_{p}\right)$ denote the coordinates of the $p$ th corner, $d$ and $\varepsilon$ are the thickness and relative permittivity of the wall, respectively $\theta$ is the tilting angle of the antenna array.

In the case of complex imaging scenes, the defocus and virtual images will appear in the imaging results if the corner of the building is regarded as a simple point target; therefore, the double reflection echo model of the corner is introduced in this paper.

4.3 The double reflection propagation paths signal model The indoor walls cause that electromagnetic waves not only travel between corners and antennas, but also reflect many times between antennas and walls, which make the received signals appear multi path echo [10]. For simplicity, the corner scattering center is regarded as the dihedral and the double reflection characteristics of the corner are the major issues that will be discussed in this paper, as shown in Fig. 13b. The double reflection echo is similar to the first-order multipath effect in the building. We assume that there are totally $K$ double reflection propagation paths in the effective range $d_{\text {finite }}$ of the corner, which are expressed as path $L_{1}, L_{2}, \cdots, L_{K}$, respectively [11]. Let $y_{J_{k}}(m, n)$ represents the double reflection echoes received by the path $L_{k}$ at the $m$ th frequency of the $n$th transceiver. Thus, the double reflection echoes received by the $n$th transceiver at the $m$ th frequency can be written as

$$
\begin{aligned}
y_{J}(m, n) & =\sum_{k=1}^{K} y_{J_{k}}(m, n) \\
& =\sum_{k=1}^{K} \sum_{p=1}^{Q} S_{p}(m, n) \mathfrak{I}\left[x_{p}, y_{p}\right] \exp \left(-j \omega_{m} \tau_{p, n}^{(k)}\right)
\end{aligned}
$$

where the function $\mathfrak{I}\left[x_{p}, y_{p}\right]$ works as an indicator function with the $p$ th corner. If the corner of the effective

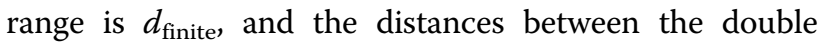
reflection point and the $p$ th corner meet $\left|x_{p}-x_{k_{1}}\right|$ $<d_{\text {finite }},\left|x_{p}-x_{k_{2}}\right|<d_{\text {finite }},\left|y_{p}-y_{k_{1}}\right|<d_{\text {finite }}$, and $\left|y_{p}-y_{k_{2}}\right|$ $<d_{\text {finite }}$ four conditions in Eq. (7), $\mathfrak{I}\left[x_{p}, y_{p}\right]=1$. The expression of the indicator function is given by

$$
\mathfrak{I}\left[x_{p}, y_{p}\right]=\left\{\begin{array}{cc}
1 & \left|x_{p}-x_{k_{1}}\right|<d_{\text {finite }}, \text { and, }\left|x_{p}-x_{k_{2}}\right|<d_{\text {finite }}, \text { and } \\
0 & \left|y_{p}-y_{k_{1}}\right|<d_{\text {finite }}, \begin{array}{c}
\text { and, },\left|y_{p}-y_{k_{2}}\right|<d_{\text {finite }} \\
\text { otherwise }
\end{array}
\end{array}\right.
$$

Other double reflections that do not satisfy this condition in Eq. (7) are not considered. The $\tau_{p, n}^{(k)}$ in Eq. (6)

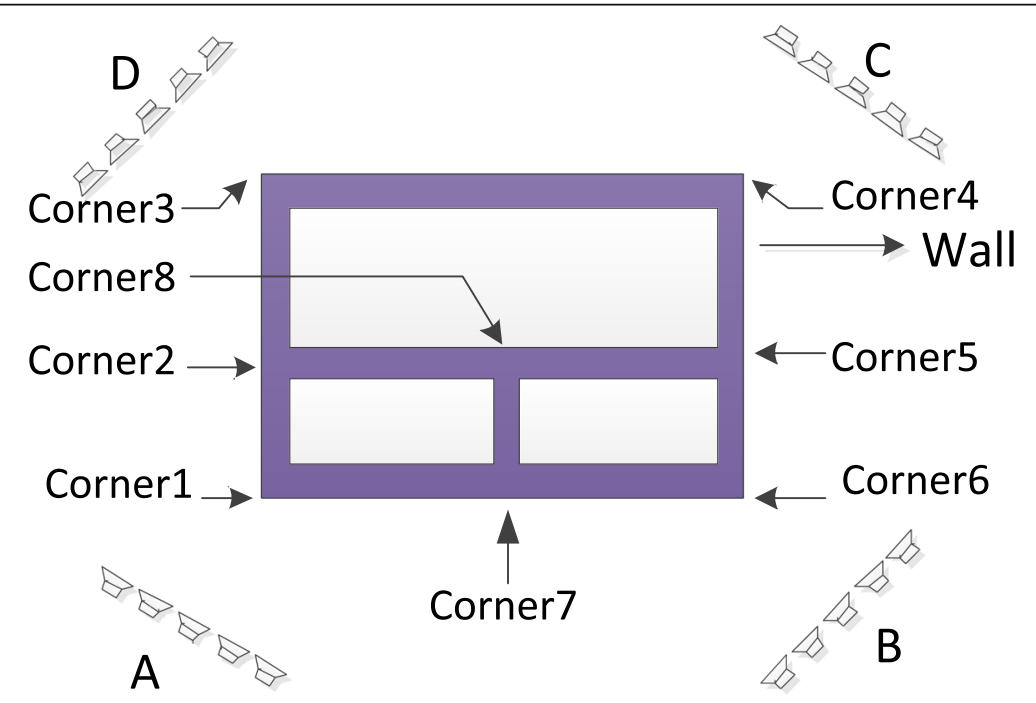

Fig. 12 Building layout 


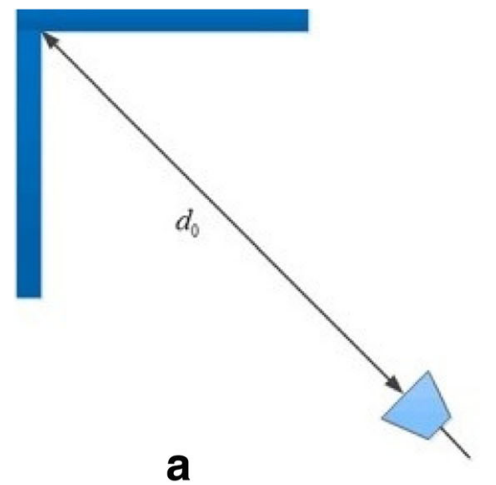
One propagation path

describes the $k$ th double reflection path traveling time from the $n$th antenna to the $p$ th corner scatterer. The electromagnetic wave propagates along this path $d_{1} \rightarrow$ $d_{2} \rightarrow d_{3}$ shows in Fig. 13c, or vice versa. The $\tau_{p, n}^{(k)}$ is given by

$$
\begin{aligned}
& \tau_{p, n}^{(k)}=\frac{d_{1}+d_{2}+d_{3}}{c}+\frac{2 d(\sqrt{\varepsilon}-1)}{c \cdot \cos \theta} \\
& =\sqrt{\left(x_{n}-x_{k_{1}}\right)^{2}+\left(y_{n}-y_{k_{1}}\right)^{2}}+\sqrt{\left(x_{k_{1}}-x_{k_{2}}\right)^{2}+\left(y_{k_{1}}-y_{k_{2}}\right)^{2}} \\
& \quad \frac{+\sqrt{\left(x_{k_{2}}-x_{n}\right)^{2}+\left(y_{k_{2}}-y_{n}\right)^{2}}}{c}+\frac{2 d(\sqrt{\varepsilon}-1)}{c \cdot \cos \theta}
\end{aligned}
$$

where $\left(x_{k_{1}}, y_{k_{1}}\right)$ and $\left(x_{k_{2}}, y_{k_{2}}\right)$ represent the coordinates of the two reflection points of the $k$ th double reflection, respectively.

Based on the above analysis, the total received signal of building corner echoes is a combination of direct path echo and the double reflection echoes returns, which is described as

$$
y(m, n)=y_{T}(m, n)+y_{J}(m, n) .
$$

\subsection{Sparse representation of the image}

In view of the corner model in the indoor environment, the direct path imaging dictionary and the double reflection imaging dictionary are constructed based on the sparse description $[10,11]$, and the virtual images are suppressed based on the non-correlation of noise in different dictionaries.

The building scene is divided into $N_{X} \times N_{Y}$ pixels. The imaging dictionary $\mathbf{A}_{T}$ corresponds to the direct transmission between the location of the antenna and the pixels in the corner of the building, which is called the direct path echo dictionary. The imaging dictionary $\mathbf{A}_{J}$ corresponds to multiple double reflection transmission between the location of the antenna and the pixels in the corner of the building, which is called the double reflection echo dictionary. The $\mathbf{A}_{T}$ denotes the $M N \times$ $N_{X} N_{Y}$ dimensional matrix and is expressed as

$$
\mathbf{A}_{T}=\left[\begin{array}{cccc}
S_{1,1}(1,1) & S_{1,2}(1,1) & \cdots & S_{N_{X}, N_{Y}}(1,1) \\
S_{1,1}(1,2) & S_{1,2}(1,2) & \cdots & S_{N_{X}, N_{Y}}(1,2) \\
\vdots & \vdots & \cdots & \vdots \\
S_{1,1}(M, N) & S_{1,2}(M, N) & \cdots & S_{N_{X}, N_{Y}}(M, N)
\end{array}\right]_{M N \times N_{X} N_{Y}}
$$

where $S_{i, j}(m, n)$ represents the direct path echo sample in Eq. (11) between the $n$th transceiver at the $m$ th frequency and the $(i, j)$ th pixel point with the scattering amplitude $A_{A_{i, j}}$ and the corresponding direct propagation path time delay $\tau_{i, j}^{(A)}$ in Eq. (12).

$$
\begin{aligned}
& S_{i, j}(m, n)=A_{A_{i, j}} \exp \left(-j \omega_{m} \tau_{i, j}^{(A)}\right), \\
& \tau_{i, j}^{(A)}=\frac{2 \sqrt{\left(x_{i}-x_{n}\right)^{2}+\left(y_{j}-y_{n}\right)^{2}}}{c}+\frac{2 d(\sqrt{\varepsilon}-1)}{c \cdot \cos \theta} .
\end{aligned}
$$

The $k$ th double reflection echo dictionary $\mathbf{A}_{J_{k}}$ is an $M N \times N_{X} N_{Y}$ dimensional matrix with the expression given by

$$
\mathbf{A}_{J_{k}}=\left[\begin{array}{cccc}
S_{J_{k}, 1,1}(1,1) & S_{I_{k}, 1,2}(1,1) & \cdots & S_{K_{k}, N_{X}, N_{Y}}(1,1) \\
S_{J_{k}, 1,1}(1,2) & S_{I_{k}, 1,2}(1,2) & \cdots & S_{J_{k}, N_{X}, N_{Y}}(1,2) \\
\vdots & \vdots & \cdots & \vdots \\
S_{J_{k}, 1,1}(M, N) & S_{J_{k}, 1,2}(M, N) & \cdots & S_{J_{k}, N_{X}, N_{Y}}(M, N)
\end{array}\right]_{M N \times N_{X} N_{Y}}
$$

where $S_{J_{k}, i, j}(m, n)$ is the $k$ th double reflection echo sample of the $m$ th frequency between the $n$th transceiver from the $(i, j)$ th pixel point in Eq. (14). $\tau_{i, j}^{(k)}$ is the corresponding double reflection propagation path delay with the same form as Eq. (8), and $A_{k}$ represents the scattering amplitude of the double reflection propagation path of the $(i, j)$ th pixel point. 


$$
\begin{aligned}
& S_{J_{k}, i, j}(m, n)=A_{k} \exp \left(-j \omega_{m} \tau_{i, j}^{(k)}\right) \\
& \tau_{i, j}^{(k)}=\sqrt{\left(x_{n}-x_{k_{i}}\right)^{2}+\left(y_{n}-y_{k_{i}}\right)^{2}}+\sqrt{\left(x_{k_{i, 1}}-x_{k_{i, 2}}\right)^{2}+\left(y_{k_{j, 1}}-y_{k_{j, 2}}\right)^{2}} \\
& \quad \frac{+\sqrt{\left(x_{k_{i, 2}}-x_{n}\right)^{2}+\left(y_{k_{j, 2}}-y_{n}\right)^{2}}}{c}+\frac{2 d(\sqrt{\varepsilon}-1)}{c \cdot \cos \theta}
\end{aligned}
$$

where $\left(x_{k_{i, 1}}, y_{k_{j, 1}}\right)$ and $\left(x_{k_{i, 2}}, y_{k_{j, 2}}\right)$ represent the coordinates of the two reflection points of the $k$ th double reflection correspond to the $\left(x_{k}, y_{k}\right)$, respectively. By stacking all the received signal $y(m, n)$ in one column vector, we obtain the radar observation vector with the following expression

$$
\mathbf{y}=[y(1,1), y(1,2), \cdots, y(M, N)]^{T} .
$$

The real imaging scenario can be estimated based on the received signal $\mathbf{y}$ which can be written as

$$
\mathbf{y}=\mathbf{A}_{T} \mathbf{x}_{T}+\sum_{k=1}^{K} \mathbf{A}_{J_{k}} \mathbf{x}_{k}
$$

where $\boldsymbol{x}_{T}$ and $\boldsymbol{x}_{k}$ are defined as the scattering coefficient vector. The multiple scene estimation images can be obtained according to multiple dictionaries. Assuming that $N_{X} N_{Y}$ is much larger than $M N$, we can conclude that $\mathbf{A}_{T}$ and $\mathbf{A}_{J_{k}}$ are only row full rank matrices. The dictionary of direct wave imaging is represented by $\mathbf{A}_{\mathrm{DPD}}$, where $\mathbf{A}_{\mathrm{DPD}}=$ $\mathbf{A}_{T}$ and $\mathbf{A}_{\mathrm{DPD}}^{+}$represents the pseudo-inverse of $\mathbf{A}_{\mathrm{DPD}}$. Multiply both sides of Eq. (17) by $\mathbf{A}_{\mathrm{DPD}}^{+}$, we can derive

$$
\begin{aligned}
\mathbf{A}_{\mathrm{DPD}}^{+} \mathbf{y}= & \mathbf{A}_{\mathrm{DPD}}^{+}\left(\mathbf{A}_{T} \boldsymbol{x}_{T}+\sum_{k=1}^{K} \mathbf{A}_{J_{k}} \boldsymbol{x}_{k}\right) \\
= & \mathbf{A}_{\mathrm{DPD}}^{+} \mathbf{A}_{T} \boldsymbol{x}_{T}+\mathbf{A}_{\mathrm{DPD}}^{+} \mathbf{A}_{J_{1}} \mathbf{x}_{1} \\
& +\mathbf{A}_{\mathrm{DPD}}^{+} \mathbf{A}_{J_{2}} \mathbf{x}_{2}+\mathbf{A}_{\mathrm{DPD}}^{+} \mathbf{A}_{J_{3}} \mathbf{x}_{3}+\cdots
\end{aligned}
$$

where $\mathbf{A}_{\mathrm{DPD}}^{+} \mathbf{A}_{T} \mathbf{x}_{T}$ has a larger value, $\mathbf{A}_{\mathrm{DPD}}^{+} \mathbf{A}_{J_{1}} \mathbf{x}_{1}$ also has a certain pixel value, the other terms are very small. Since the corner of the building is regarded as an extended target, not a scatter point target, $\mathbf{A}_{\mathrm{DPD}}^{+} \mathbf{A}_{J_{1}} \mathbf{x}_{1}$ represents the corresponding multipath ghost image to path $L_{1}$. Similarly, $\mathbf{A}_{\mathrm{DPD}}^{+} \mathbf{A}_{J_{2}} \mathbf{x}_{2}$ and $\mathbf{A}_{\mathrm{DPD}}^{+} \mathbf{A}_{J_{3}} \mathbf{x}_{3}$ are respectively the multipath ghost images related to path $L_{2}$ and path $L_{3}$. Assuming $\boldsymbol{\Phi}_{y}$ is the measurement matrix, the scene imaging results of the direct path imaging dictionary can be obtained by solving sparse signal recovery problem in Eq. (19) [12].

$$
\mathbf{x}_{T}=\arg \min _{x}\left\|\mathbf{x}_{T}\right\|_{0} \quad \text { s.t }\left\|\hat{\mathbf{y}}-\boldsymbol{\Phi}_{y} \mathbf{A}_{\mathrm{DPD}} \mathbf{x}_{T}\right\|_{2}^{2} \leq \varepsilon_{0}
$$

Let $\mathbf{A}_{\mathrm{MPD}}^{(k)}$ be the $k$ th double reflection imaging dictionary, where $\mathbf{A}_{\mathrm{MPD}}^{(k)}=\mathbf{A}_{J_{k}}$, and $\mathbf{A}_{\mathrm{MPD}}^{(k)+}$ represents the pseudo-inverse. Multiply both sides of Eq. (17) by $\mathbf{A}_{\mathrm{DPD}}^{+}$, we can derive

$$
\begin{aligned}
\mathbf{A}_{\mathrm{MPD}}^{(k)+} \mathbf{y}= & \mathbf{A}_{\mathrm{MPD}}^{(k)+} \mathbf{A}_{T} \mathbf{x}_{T}+\mathbf{A}_{\mathrm{MPD}}^{(k)+} \mathbf{A}_{J_{1}} \mathbf{x}_{1} \\
& +\mathbf{A}_{\mathrm{MPD}}^{(k)+} \mathbf{A}_{J_{2}} \mathbf{x}_{2}+\mathbf{A}_{\mathrm{MPD}}^{(k)+} \mathbf{A}_{J_{3}} \mathbf{x}_{3}+\cdots
\end{aligned}
$$

where $\mathbf{A}_{\mathrm{MPD}}^{(k)+} \mathbf{A}_{J_{k}} \mathbf{x}_{k}$ represents the corresponding multipath image to double reflection path $L_{k}$. The scene imaging results of the $k$ th double reflection imaging dictionary can be obtained by solving sparse signal recovery problem in Eq. (21).

$$
\mathbf{x}_{k}=\arg \min _{x}\left\|\mathbf{x}_{k}\right\|_{0} \quad \text { s.t }\left\|\hat{\mathbf{y}}-\Phi_{y} \mathbf{A}_{\mathrm{MPD}}^{(k)} \mathbf{x}_{k}\right\|_{2}^{2} \leq \varepsilon_{1}
$$

OMP algorithm is used to solve the problem of sparse signal recovery of Eqs. (19) and (21).

\subsection{Image fusion of multiple dictionaries}

Based on the above analysis, both the estimated image derived from the direct path imaging dictionary and the estimated images produced by the double reflection imaging dictionaries contain the target image of interest. Moreover, the elements will form a highlight focused region located at the position of the true target, and the noise in these images will not overlap [15].

In order to extract the building corner and delete the noise, we fuse these estimated images together. Based on the above features, these images are fused by a noncoherent image fusion method to produce a no-false image. To be clear, for the image $\mathbf{x}_{T}$ and $\mathbf{x}_{1}$ of this example, only the path $L_{1}$ is considered, we derive

$$
\begin{aligned}
\boldsymbol{x}_{f, 1}= & \left|\mathbf{x}_{T}\right| \cdot\left|\mathbf{x}_{1}\right|=\left|\mathbf{A}_{\mathrm{DPD}}^{+} \mathbf{A}_{T} \mathbf{x}_{T}+\mathbf{A}_{\mathrm{DPD}}^{+} \mathbf{A}_{J_{1}} \mathbf{x}_{1}\right| \cdot\left|\mathbf{A}_{\mathrm{MPD}}^{(1)+} \mathbf{A}_{T} \mathbf{x}_{T}+\mathbf{A}_{\mathrm{MPD}}^{(1)+} \mathbf{A}_{I_{1}} \mathbf{x}_{1}\right| \\
= & \left|\mathbf{A}_{\mathrm{DPD}}^{+} \mathbf{A}_{T} \mathbf{x}_{T}\right| \cdot\left|\mathbf{A}_{\mathrm{MPD}}^{(1)+} \mathbf{A}_{T} \mathbf{x}_{T}\right|+\left|\mathbf{A}_{\mathrm{DPD}}^{+} \mathbf{A}_{J_{1}} \mathbf{x}_{1}\right| \cdot\left|\mathbf{A}_{\mathrm{MPD}}^{(1)+} \mathbf{A}_{I_{1}} \mathbf{x}_{1}\right|+ \\
& \left|\mathbf{A}_{\mathrm{DPD}}^{+} \mathbf{A}_{T} \mathbf{x}_{T}\right| \cdot\left|\mathbf{A}_{\mathrm{MPD}}^{(1)+} \mathbf{A}_{J_{1}} \mathbf{x}_{1}\right|+\left|\mathbf{A}_{\mathrm{DPD}}^{+} \mathbf{A}_{I_{1}} \mathbf{x}_{1}\right| \cdot\left|\mathbf{A}_{\mathrm{MPD}}^{(1)+} \mathbf{A}_{T} \mathbf{x}_{T}\right|
\end{aligned}
$$

where $\boldsymbol{x}_{f, 1}$ denotes the fusion result of image $\mathbf{x}_{T}$ and $\mathbf{x}_{1}$, $|\cdot|$ represents the absolute value operation. By inspecting Eq. (22), we could observe that $\mathbf{A}_{\mathrm{DPD}}^{+} \mathbf{A}_{T} \mathbf{x}_{T}$ and $\mathbf{A}_{\mathrm{MPD}}^{(1)+} \mathbf{A}_{J_{1}}$ $\mathbf{x}_{1}$ are the target images derived from the direct path imaging dictionary and the first double reflection imaging dictionary, respectively. $\mathbf{A}_{\mathrm{DPD}}^{+} \mathbf{A}_{J_{1}} \mathbf{x}_{1}$ and $\mathbf{A}_{\mathrm{MPD}}^{(1)+} \mathbf{A}_{T} \mathbf{x}_{T}$ are the fake images and noise with respect to the direct path imaging dictionary and the first double reflection imaging dictionary, respectively. It is clear that the third item $\left|\mathbf{A}_{\mathrm{DPD}}^{+} \mathbf{A}_{T} \mathbf{x}_{T}\right| \cdot\left|\mathbf{A}_{\mathrm{MPD}}^{(1)+} \mathbf{A}_{J_{1}} \mathbf{x}_{1}\right|$ represents the genuine target image, and the other three items are approximated to zeros. In the same way, we obtain $\mathbf{x}_{f, k}$ which denotes the fusion result of image $\mathbf{x}_{T}$ and $\mathbf{x}_{k}$ from the path $L_{k}$ of the double reflection echoes in the corner. 
Three double reflections are taken into account, and the expression of the fused image $\boldsymbol{x}_{f}$ is described as below

$$
\begin{aligned}
\mathbf{x}_{f}= & \mathbf{x}_{f 1} \cdot \mathbf{x}_{f 2} \cdot \mathbf{x}_{f 3} \approx\left|\mathbf{A}_{\mathrm{DPD}}^{+} \mathbf{A}_{T} \mathbf{x}_{T}\right|^{3} \\
& \cdot\left|\mathbf{A}_{\mathrm{MPD}}^{(1)+} \mathbf{A}_{J_{1}} \mathbf{x}_{1}\right| \cdot\left|\mathbf{A}_{\mathrm{MPD}}^{(2)+} \mathbf{A}_{J_{2}} \mathbf{x}_{2}\right| \\
& \cdot\left|\mathbf{A}_{\mathrm{MPD}}^{(3)+} \mathbf{A}_{J_{3}} \mathbf{x}_{3}\right|
\end{aligned}
$$

where $\mathbf{x}_{f}$ represents the fusion result of image $\mathbf{x}_{T}, \mathbf{x}_{J_{1}}$, $\mathbf{x}_{I_{2}}$, and $\mathbf{x}_{J_{3}}$. When radar detection is performed around the building, it is necessary to incoherent fusion of images from multiple-viewpoint, we have

$$
\mathbf{x}_{f \text {-sum }}=\mathbf{x}_{f-A}+\mathbf{x}_{f-B}+\mathbf{x}_{f-C}+\mathbf{x}_{f-D}
$$

where $\mathbf{x}_{f \text { - sum }}$ denotes the fusion result of image $\mathbf{x}_{f-A}, \mathbf{x}_{f \text { - }}$ ${ }_{B}, \mathbf{x}_{f-C}$, and $\mathbf{x}_{f-D}$, which are respectively the image derived from the four single-location image, as shown in Fig. 12.

\section{Abbreviations}

ENT: Entropy of the image; OMP: Orthogonal matching pursuit; SCR: Signalto-clutter ratio; TCR: Target-to-clutter ratio; TWRI: Through-the-wall radar imaging

\section{Acknowledgements}

The authors would like to thank Tingfa Xu for the support.

\section{Authors' contributions}

HD and TX conceived of the building feature extraction method based on double reflection imaging dictionary. All authors read and approved the final manuscript.

\section{Funding}

This work was supported by the Major Science Instrument Program of the National Natural Science Foundation of China under grant 61527802. This work was supported by the Major Science and Technology Foundation of Guangxi Province under grant AA17204093.

Availability of data and materials

All data are fully available without restriction.

\section{Competing interests}

The authors declare that they have no competing interests.

\section{Author details \\ 'Key Laboratory of Photoelectronic Imaging Technology and System, Beijing Institute of Technology, Beijing, China. ${ }^{2}$ School of Information and Communication, Guilin University of Electronic Technology (GUET), Guilin, China}

Received: 6 May 2019 Accepted: 25 July 2019

Published online: 11 October 2019

\section{References}

1. A. Bouzerdoum, V.H. Tang, S.L. Phung, in IEEE Radar Conference (RadarConf) A low-rank and jointly-sparse approach for multi polarization through-wall radar imaging (Seattle, 2017), pp. 0263-0268

2. J. Liu, L.j. Kong, X. Yang, Refraction angle approximation algorithm for wall compensation in TWRI. IEEE Geosci. Remote Sens. Lett. 13(7), 943-946 (2016)

3. B. Yektakhah, K. Sarabandi, All-directions through-the wall radar imaging using a small number of moving transceivers. IEEE Trans. Geosci. Remote Sens. 54(11), 6415-6428 (2016)

4. P.C. Chang, Near Zone Radar Imaging and Feature Capture of Building Interiors (The Ohio State University, Columbus, 2008)

5. Y. Jia, X. Zhong, J. Liu, Y. Guo, Single-side two-location spotlight imaging for building based on MIMO through-wall-radar. Sensors 16(9), 1441 (2016)
6. E. Lagunas, M.G. Amin, F. Ahmad, M. Najar, Determining building interior structures using compressive sensing. J. Electron. Imaging 22(2), 381-388 (2013) [55]

7. E. Lagunas, M.G. Amin, F. Ahmad, M. Najar, Pattern matching for building feature extraction [J]. IEEE Geosci. Remote Sens. Lett. 11(12), 2193-2197 (2014)

8. B. Chen, T. Jin, B. Lu, Z. Zhou, in IET International Radar Conference. Polarimetric characteristics analysis of interior structures of a building in through-the-wall radar imaging (2013), p. 0229

9. H. Liu, B. Jiu, F. Li, Y. Wang, Attributed scattering center extraction algorithm based on sparse representation with dictionary refinement. IEEE Trans. Antennas Propag. 65(5), 2604-2614 (2017)

10. M. Leigsnering, F. Ahmad, M. Amin, A. Zoubir, Parametric dictionary learning for sparsity-based TWRI in multipath environments. IEEE Trans. Aerosp. Electron. Syst. 52(2), 532-547 (2016)

11. P.C. Chang, R.J. Burkholder, J.L. Volakis, High-frequency EM characterization of through-wall building imaging. IEEE Trans. Geosci. Remote Sens. 47(5), 1375-1387 (2009)

12. A. Hoorfar, W. Zhang, in Applied Computational Electromagnetics. MIMO radar imaging of targets behind multilayered walls using compressive sensing (2015), pp. 1-2

13. S. Guo, G. Cui, M. Wang, L. Kong, X. Yang, Similarity-based multipath suppression algorithm for through-wall imaging radar. IET Radar Sonar Navig. 11(7), 1041-1050 (2017)

14. X. Chen, W. Chen, Double-layer fuzzy fusion for multiview through-wall radar images. IEEE Geosci. Remote Sens. Lett. 12(10), 2075-2079 (2015)

15. G. Cui, L. Kong, X. Yang, Reconstruction filter design for stepped-frequency continuous wave. IEEE Trans. Signal Process. 60(8), 4421-4426 (2012)

\section{Publisher's Note}

Springer Nature remains neutral with regard to jurisdictional claims in published maps and institutional affiliations.

\section{Submit your manuscript to a SpringerOpen ${ }^{\odot}$ journal and benefit from:}

- Convenient online submission

- Rigorous peer review

- Open access: articles freely available online

- High visibility within the field

- Retaining the copyright to your article

Submit your next manuscript at $\boldsymbol{\nabla}$ springeropen.com 